\title{
Melhoramento cognitivo farmacológico: futuro promissor? Ou futuro inevitável?
}

\author{
Rafael Cardoso Oliveira ${ }^{1}$, Rui Nunes ${ }^{1}$ \\ 1. Universidade do Porto, Porto, Portugal.
}

\begin{abstract}
Resumo
Funções cognitivas são alvo frequente de curiosidade, estudo e reflexão, pois são estruturantes para o ser o humano tal como o conhecemos. Compreender os limites cognitivos, os processos que os originam e a forma de os ultrapassar é, por isso, forma de entender a condição humana e de perceber as consequências da potencial manipulação da cognição. Nos últimos anos tem havido interesse crescente no melhoramento cognitivo mediante o uso de fármacos, e com ele têm surgido diversos e importantes dilemas éticos, médicos e legais. O objetivo deste ensaio é refletir sobre os problemas éticos levantados. Pode-se concluir que o melhoramento cognitivo farmacológico tem de ser mais bem estudado no nível farmacodinâmico para que sua aplicação - regulada e em contextos específicos - possa beneficiar indivíduos e sociedade, não pondo em causa a autenticidade da condição humana.
\end{abstract}

Palavras-chave: Melhoramento biomédico. Ética médica. Nootrópicos.

\section{Resumen}

Mejoramiento cognitivo farmacológico: ¿Un futuro prometedor? ¿O un futuro inevitable?

Las funciones cognitivas humanas son objeto frecuente de curiosidad, estudio y reflexión, ya que estructuran el ser humano tal como lo conocemos. Comprender los límites cognitivos, los procesos que los originan y la forma de superarlos es, por lo tanto, una forma de entender la condición humana y percibir las consecuencias de la potencial manipulación de la cognición. En los últimos años ha habido un interés creciente en el mejoramiento cognitivo a través del uso de productos farmacéuticos, y con ello han surgido una serie de importantes dilemas éticos, médicos y legales. El propósito de este ensayo es reflexionar sobre los problemas éticos planteados. Se puede concluir que el mejoramiento cognitivo farmacológico debe estudiarse mejor en el ámbito farmacodinámico, de modo que su aplicación, regulada y en contextos específicos, pueda beneficiar a individuos y a la sociedad sin comprometer la autenticidad de la condición humana.

Palabras clave: Refuerzo biomédico. Ética médica. Nootrópicos.

\section{Abstract}

Pharmacological cognitive enhancement: a promising or an inevitable future?

Human cognitive functions are often targets of curiosity, study and reflections, since they are essential for human beings. Understanding our cognitive boundaries, the processes that originate them, and how to overcome them means comprehending the human condition and the consequences of manipulating cognition. In recent years, growing interest has been observed in cognitive enhancement with the help of drugs, resulting in several important ethical, medical, and legal dilemmas. This article analyzed the ethical issues involved in this process and concluded that pharmacological cognitive enhancement needs further studies at the pharmacodynamic level, so that its application - regulated and in specific contexts - can benefit individuals and society, without undermining the authenticity of the human condition.

Keywords: Biomedical enhancement. Ethics, medical. Nootropic agents. 
O melhoramento cognitivo farmacológico (MCF) pode ser descrito como o consumo por indivíduos saudáveis de fármacos sujeitos a prescrição médica para aumento da cognição. O MCF e todas as suas vantagens e problemas tem se alastrado enquanto prática e tema de debate neuroético. O número de artigos científicos disponíveis sobre o assunto passou de 1.300 entre 1980 e 2000 para cerca de 17 mil entre 2000 e $2017^{1}$, e também os meios de comunicação têm muito mais intensamente reportado esta prática. O objetivo deste artigo é apresentar os vários dilemas éticos e neuroéticos que advêm do MCF, bem como as várias vantagens e limitações deste procedimento no indivíduo e no contexto social em que se insere.

\section{Método}

Este ensaio se fundamentou em pesquisa bibliográfica realizada na base de dados PubMed, usando as palavras-chave "cognitive enhancement", "ethics" e "pharmacological cognitive enhancement". Foram incluídos artigos em inglês e português publicados nos últimos oito anos e cujo texto completo estivesse disponível sem custos. Foram ainda incorporados, após pesquisa manual, alguns trabalhos científicos considerados relevantes e que constavam das referências dos artigos selecionados. Excluíram-se os artigos cujo abstract ou corpo do texto, após análise, divergiam demasiadamente da problemática em apreço.

\section{Melhoramento cognitivo e}

\section{farmacológico}

O melhoramento cognitivo ( $M C$ ) é tema recente no campo da neuroética, que estuda a ética da neurociência e a neurociência da ética ${ }^{2}$. A definição de $\mathrm{MC}$ não se encontra absolutamente estabelecida, e não é claro se "melhoramento" e "tratamento" podem ser distinguidos ${ }^{3}$. Contudo, as formulações apresentadas a seguir são as mais comuns. Primeira definição: o $\mathrm{MC}$ corresponde à extensão ou amplificação de capacidades cognitivas básicas mediante o melhoramento de sistemas de processamento de informação por mecanismos internos ou externos ao indivíduo ${ }^{3,4}$. Segunda definição: $M C$ é qualquer mudança na biologia ou psicologia do indivíduo que aumenta a probabilidade de este ter melhor qualidade de vida nas circunstâncias em que está inserido ${ }^{5}$.

Vários especialistas em bioética sugerem ainda uma terceira conceituação: $M C$ será qualquer intervenção nas capacidades cognitivas que vai além do estritamente necessário à saúde do indivíduo ${ }^{6,7}$. Outra definição, mais generalista, será a proposta por Franke, Lieb e Hildt ${ }^{8}$, apoiada por outros autores: MC é o uso, sem necessidade médica e por parte de indivíduos saudáveis, de fármacos, biotecnologia apropriada ou outros meios para aumentar funções cognitivas como memória, concentração ou vigilância ${ }^{9,10}$.

Além destas definições aparentemente divergentes, é também díspar o limiar de "melhoramento": pode ser considerado como qualquer aumento de determinada capacidade cognitiva relativamente a um nível basal do indivíduo e que the possa aumentar as probabilidades de ter qualidade de vida melhor ${ }^{11}$. Nesta definição, o "melhoramento" ocorre independentemente do nível basal de funcionalidade de dada capacidade ${ }^{5}$. Alternativamente, há definições mais restritivas, as quais assumem que uma capacidade cognitiva está melhorada se está aumentada para além de um limite assumido como normal, ou então para além do limite mínimo de correção de patologia ${ }^{11}$.

Apesar de tudo, podem-se encontrar pontos comuns a estas definições e, para os fins a que este ensaio se propõe, $\mathrm{MC}$ será considerado como qualquer melhoria de determinada capacidade cognitiva de um indivíduo saudável e que provavelmente melhorará sua qualidade de vida. Há várias formas conhecidas e utilizadas para melhorar a cognição dos indivíduos, entre as quais se destacam o MCF e o melhoramento cognitivo não farmacológico (MCNF).

O MCNF está associado à ideia de que as capacidades cognitivas podem ser mantidas ou até melhoradas com nutrição adequada, atividade física regular, higiene do sono e descanso apropriados, além de várias formas de meditação e yoga ou mesmo com o uso de mnemónicas e outros sistemas de memorização ${ }^{12,13}$. Estão ainda associados a este conceito, por exemplo, o uso de outros métodos não invasivos, como o treino computadorizado (por meio de jogos apropriados) ${ }^{13}$. Há também outros métodos, como a estimulação elétrica do encéfalo por meio de Transcranial Direct Current Stimulation, 
Transcranial Magnetic Stimulation, Direct Vagus Nerve Stimulation ou Deep Brain Stimulation, sendo esta última a técnica mais invasiva ${ }^{13}$.

O MCF é uma das formas mais discutidas atualmente na comunidade científica ${ }^{14}$ e nos meios de comunicação ${ }^{15}$, e seu impacto e as problemáticas neuroéticas que apresenta são mais evidentes do que o melhoramento cognitivo não associado a fármacos. Tendo em conta as citadas definições, pode-se assumir o MCF como o uso de fármacos por parte de indivíduos saudáveis com vistas ao aumento de determinadas capacidades cognitivas sem que haja necessidade médica.

\section{Principais fármacos usados no melhoramento cognitivo farmacológico}

Os fármacos comumente associados ao MCF são o metilfenidato (Ritalina), compostos de anfetamina-dextroanfetamina/sais de várias anfetaminas (Adderall) e o modafinil (Provigil) ${ }^{3,16}$. O metilfenidato é um inibidor da recaptação sináptica da dopamina e noradrenalina, usado no tratamento da perturbação de hiperactividade e défice de atenção (PHDA). A anfetamina-dextroanfetamina aumenta a liberação de dopamina, e é também usada na PHDA. Por sua vez, o modafinil é utilizado no tratamento da narcolepsia e de doenças do sono, e é um fármaco com múltiplos mecanismos de ação ${ }^{3}$.

Além destes há outras substâncias, como donepezil, galantamina e rivastigmina (entre outros agonistas da dopamina), cujo efeito no MCF é menos notório, mas também menos estudado ${ }^{11}$. Certos estudos admitem um efeito nulo, ou mesmo deletério, por parte destes últimos no $\mathrm{MCF}^{11,17-19}$. Há, no entanto, que referir que a maior parte da literatura disponível, mesmo em relação aos nootrópicos principais, revela resultados díspares, desde parcos incrementos nas funções cognitivas pesquisadas até efeitos negativos nessas ou em outras funções ${ }^{3,14}$.

Outros fármacos utilizados para aumentar o desempenho, embora em âmbito normalmente diferente, são os $\beta$-bloqueadores, utilizados por vários músicos para minorar tremores durante as intervenções musicais ${ }^{3}$. Todos estes fármacos, pelo seu uso enquanto potenciais impulsionadores da cognição em indivíduos saudáveis, podem também ser designados como nootrópicos.

\section{Enquadramento social do melhoramento cognitivo} farmacológico

O uso dos nootrópicos por parte do público em geral reflete o desejo dos indivíduos (ou a pressão exercida sobre eles) de melhorar sua cognição, mesmo sendo saudáveis ${ }^{19}$, e terá várias motivações, como o estresse associado ao trabalho ou a competitividade no meio académico e científico ${ }^{19,20}$. Apesar disto, várias pesquisas indicam que o MCF entre estudantes universitários não é a regra ${ }^{10,21-25}$. Note-se também que o meio estudantil inclui crianças e adolescentes, e estes podem ser sujeitos a pressões diversas para encetar programas de $\mathrm{MCF}^{19}$.

O MCF pode apresentar benefícios em profissões com níveis altos de pressão ou risco, ou naquelas em que os profissionais necessitem de maior capacidade de adaptação e aprendizagem ${ }^{19}$. Hodiernamente, em vários ambientes profissionais espera-se que os trabalhadores sejam mais eficientes durante mais tempo. Além de se exigir deles mais flexibilidade, motivação e produtividade, o ambiente competitivo e o desejo de sucesso são constantes ${ }^{19,26}$. O uso de nootrópicos é, assim, frequente em profissões mais exigentes ${ }^{19,26}$. Por outro lado, mesmo o desempenho de trabalhadores com défice de horas de sono ou descanso poderá vir a ser melhorado pelo MCF, aumentando sua eficácia, seu rendimento e a segurança no trabalho ${ }^{19,26}$.

O desejo de melhoria cognitiva e do desempenho não está, no entanto, limitado a estudantes e profissionais. Há também indivíduos (designados "psiconautas") que usam fármacos com propriedades alteradoras da cognição e que regulam o uso de várias drogas, registando suas experiências ${ }^{19}$. A par disto, o consumo de microdoses de drogas com efeitos psicadélicos, como dietilamida do ácido lisérgico (LSD), a psilocibina (presente em alguns cogumelos) ou a mescalina, é cada vez mais frequente ${ }^{19}$ e tem o objetivo de melhorar funções cognitivas como a criatividade ou a percepção, com fins profissionais ou recreativos.

Contudo, é importante notar que uma das limitações da compreensão e do uso do MCF (e dos vários nootrópicos) é a falta de estudos para cada fármaco, bem como a falta de informação sobre seus efeitos secundários e, principalmente, em longo prazo ${ }^{11,19}$. 
Isto levanta várias questões quanto à segurança e pertinência do MCF, que serão brevemente explanadas em seguida, a par de outras problemáticas neuroéticas importantes inerentes ao uso de nootrópicos nos contextos abordados.

\section{Questões neuroéticas essenciais do melhoramento cognitivo farmacológico}

Com a disseminação de seu uso e o crescente interesse em seu estudo, o MCF tem levantado questões neuroéticas controversas ${ }^{3,10,12}$. De modo a simplificar sua explicação, estas, associadas às suas vantagens e desvantagens, serão apresentadas em subseções.

\section{Desconhecimento da eficácia e dos efeitos em longo prazo}

Uma das principais limitações do MCF é a falta de conhecimento quanto aos seus efeitos sobre o cérebro (e a restante fisiologia) associados ao consumo de nootrópicos ${ }^{12,19}$. Note-se que estudos reportam melhorias cognitivas em indivíduos saudáveis, mas apenas como efeitos colaterais não esperados ${ }^{27}$, pois esses estudos tinham outros objetivos. Desconhece-se se os nootrópicos serão eficazes no MCF, e principalmente o seu perfil de segurança e efeitos colaterais em indivíduos saudáveis ${ }^{11,12}$, visto que são estudados em contextos de doença ou défice como forma de prevenção, diagnóstico ou tratamento ${ }^{12}$.

O consumo continuado de nootrópicos (ou fármacos psicotrópicos) poderá levar a alterações nos circuitos neuronais ou das sinapses, com efeitos desconhecidos e que poderão ser deletérios em longo prazo ${ }^{3,12}$. Há registo de efeitos negativos do metilfenidato em estudantes saudáveis quanto à qualidade do sono, assim como um aumento de sintomatologia depressiva nestes indivíduos ${ }^{3}$. Tudo isto é particularmente importante no caso do seu uso (por pressão parental ou de professores) em crianças e adolescentes ${ }^{12,19,28}$, cujos encéfalos ainda se encontram em desenvolvimento.

O desconhecimento dos efeitos e riscos associados aos nootrópicos poderá justificar a limitação do seu uso a períodos curtos ou a circunstâncias específicas, e apenas mediante razões válidas ${ }^{12,29}$. Aplicam-se aqui os princípios básicos da não maleficência e da beneficência.

\section{Possiveis modificações na personalidade,} individualidade e autenticidade

O consumo de nootrópicos tem acarretado crescente preocupação ética devido a possível modificação da personalidade dos indivíduos ${ }^{3}$, podendo alterar características tidas como essenciais ao ser humano ${ }^{3,30}$. Por exemplo, questiona-se se o MCF não irá alterar tendências naturais e fazer crer que as emoções deverão ser também modificadas ou moduladas pelo $\mathrm{MCF}^{3,16}$. Questiona-se mesmo se o consumo continuado destes fármacos não levará os indivíduos a se perceberem como algo diferente do ser humano, e a subvalorizar algumas características da própria condição humana ${ }^{3,31}$.

No caso de crianças e adolescentes, há implicações biológicas e psicológicas importantes, valendo a pena salientá-las. Biologicamente, o impacto desconhecido do MCF nos encéfalos em desenvolvimento é ainda mais preocupante do que nos indivíduos adultos, e as suas capacidades de alteração poderão ser exacerbadas, levando a transformações na individualidade, personalidade e até na autenticidade (e no sentimento de autenticidade) dos indivíduos ${ }^{3}$. Psicologicamente, é sabido que crianças e indivíduos mais jovens atribuem parte do seu comportamento (principalmente o mau comportamento) a picos de abstinência destes fármacos, o que levanta questões imediatas (e em longo prazo) quanto à percepção de moralidade e ética por parte destes indivíduos ${ }^{3,28,32,33}$. Isto reforça as questões sobre a individualidade e autenticidade em epígrafe.

\section{Imparidade e injustiça no acesso aos nootrópicos}

O acesso aos nootrópicos pode contribuir para o aumento das disparidades sociais ${ }^{34-36}$. Todavia, se esse acesso não depender da capacidade financeira dos indivíduos ou for programado para favorecer os mais necessitados, o efeito poderá ser diametralmente oposto, diminuindo as disparidades $^{34,35,37}$. Priorizar e apoiar o uso de MCF em indivíduos desfavorecidos, limitando-se o acesso à medida que os favorecimentos sociais aumentam, poderá ser uma forma de melhorar o estado social ${ }^{3}$. Pequenos ganhos cognitivos podem melhorar significativamente os resultados académicos, por exemplo $^{38}$, e é sabido que o maior acesso à graduação e pós-graduação por estudantes oriundos de famílias mais favorecidas já é um problema em si. 
O impacto social do melhoramento cognitivo de vários indivíduos poderá ser significativo, diminuindo as dificuldades de aprendizagem e mesmo a incidência de atraso mental. Esta melhoria poderia mesmo ter efeitos importantíssimos na economia, na tecnologia e na cultura, dado o aumento no quociente de inteligência (Q.I.) da população em geral ${ }^{27}$. Há quem advogue que uma subida tão ligeira quanto apenas $3 \%$ no Q.I. mundial médio poderia reduzir a taxa de pobreza em $25 \%^{3,39}$, produzir um ganho económico de 165 a 195 bilhões de dólares e um crescimento de 1,5\% no PIB ${ }^{7}$.

\section{Coerção e soft peer pressure}

Embora a coerção para o consumo de nootrópicos seja reportada como de pouca preocupação para a população estudantil ${ }^{3,21,40,41}$, o mesmo poderá não ser verdade para outras faixas populacionais. O caso mais paradigmático será o MCF em crianças e adolescentes por pressão parental ou de professores ${ }^{3}$, situação em que, como anteriormente descrito, os efeitos potencialmente nefastos do MCF se poderão fazer notar mais acentuadamente. Não se trata de uma raridade, como exemplifica uma pesquisa da Nature, em que $33 \%$ dos inquiridos admitiram que se sentiriam pressionados a dar nootrópicos aos filhos caso outras crianças os consumissem na escola ${ }^{42}$.

A pressão social indireta por parte dos pares ("soft peer pressure") parece preocupar mais os estudantes do que a coerção ${ }^{3,21}$. Esta pressão consiste na percepção, por parte do estudante, de que um grupo a que pertence está a consumir nootrópicos visando o MCF e o estudante não. $O$ consumo de nootrópicos e o MCF estão comumente associados às fraternities e sororities estadunidenses ${ }^{10} \mathrm{e}$ a grupos de amigos comuns exatamente por esse motivo ${ }^{10}$. Note-se, no entanto, que esta soft peer pressure poderá condicionar o comportamento oposto em sociedades ou grupos que culturalmente vêem o MCF como algo a evitar ${ }^{21}$, muitas das vezes usando do argumento da ilegalidade do consumo destas drogas para fins não terapêuticos ${ }^{43}$.

A coerção e a soft peer pressure fazem-se também sentir nos locais de trabalho (principalmente nos mais exigentes), onde cada vez mais se espera que os trabalhadores sejam mais eficazes e produtivos. A diminuição das falhas dependentes do fator humano será uma vantagem possível do próprio $\mathrm{MCF}^{26}$, mas poderá rapidamente tornar-se exigência dos empregadores ou mesmo do Estado $^{26,31}$. A própria comunidade científica não está imune a este uso: segundo Mohamed $^{3}$, em 2008 um questionário da Nature reportou que, entre 1.400 membros saudáveis da comunidade científica de 60 países, 1 em cada 5 admitiu usar nootrópicos para MCF, sendo o metilfenidato e o modafinil os mais utilizados (por 62 e $44 \%$ dos visados, respectivamente). Alguns autores consideram, contudo, arriscado aceitar o MCF como normal no seio das instituições e na sociedade ${ }^{21}$.

\section{Trade-off cognitivo}

$O$ efeito potenciador de vários fármacos do MCF parece depender do nível de cognição e capacidade basal dos indivíduos ${ }^{3}$, mas, além disto, alguns estudos evidenciam a hipótese de o "melhoramento" ser apenas um fenómeno de trade-off (ou troca recíproca), em que determinada capacidade cognitiva é melhorada mas, em troca, outra capacidade ficaria diminuída ${ }^{3,11}$. Estudo recente em crianças diagnosticadas com PHDA evidenciou que o consumo de metilfenidato aumentava o número de erros no teste Wisconsin Card Sorting, que requer flexibilidade cognitiva e capacidade de adaptação ${ }^{44}$.

Desta forma, será indispensável estudar o tema e perceber se o melhoramento induzido por fármacos de uma capacidade cognitiva é apenas conseguido prejudicando outra. Alguns estudos sugerem que há este trade-off cognitivo entre, por exemplo, a estabilidade e a flexibilidade da memória em longo prazo, a estabilidade e a flexibilidade da memória de trabalho em curto prazo, a própria memória em longo prazo e a memória de trabalho ou entre a cognição em si e a emoção e o humor ${ }^{11}$. Esta troca pode levantar problemas quanto à liberdade cognitiva ${ }^{13,26}$ das pessoas, que em circunstâncias várias se poderão ver coagidas ao MCF, como explanado. A liberdade cognitiva não se encontra, neste momento, legalmente protegida ${ }^{26}$.

\section{Uso no meío académico, justiça competitiva e valorização dos resultados}

Conforme descrito anteriormente, o uso do MCF está largamente associado à comunidade académica, principalmente nos Estados Unidos, onde a percentagem de uso de fármacos sem 
prescrição médica e com vistas ao MCF pode ir dos 5 aos $35 \%^{10,45}$, dependendo da universidade, sendo particularmente alta nas fraternities e sororities a elas associadas ${ }^{10}$. Estudos indicam que em algumas fraternities o uso de MCF pode chegar aos $55 \%{ }^{21}$. Na União Europeia, estima-se que o MCF seja adoptado por entre 0,8 e $16 \%$ dos estudantes universitários, dependendo do país, da universidade e do fármaco ${ }^{10,43,46}$. Note-se, porém, que é difícil determinar o MCF nas universidades europeias, uma vez que as amostras, os métodos e o desenho dos estudos são bastante díspares ${ }^{10,21}$.

Importa reforçar que os estudantes universitários referem o ambiente competitivo e estressante e a sensação de concentração e vigilância oferecida pelos nootrópicos como os principais motivos para o seu consumo ${ }^{19}$. Apesar disto, é clara a distinção traçada - principalmente no contexto europeu ${ }^{10,46}$ - entre os estimulantes nootrópicos de acesso livre (como café, comprimidos de café e bebidas energéticas) e os nootrópicos sujeitos a prescrição ${ }^{21}$. Os primeiros são muito mais prevalentes, consumidos diariamente pela maioria dos estudantes ${ }^{21}$, e o estigma social é descrito como inexistente para este tipo de uso ${ }^{21,31}$. Os nootrópicos sujeitos a prescrição, por outro lado, são mais mal vistos e, por vezes, discriminados por serem fenómeno indesejado típico das universidades estadunidenses ${ }^{10,21}$.

O aumento reportado do consumo de nootrópicos no meio académico ${ }^{3}$ tem ressaltado as problemáticas da justiça competitiva entre os estudantes e mesmo o valor dos resultados que estes obtêm se optarem pelo MCF. Em meio universitário cada vez mais competitivo, há quem compare o MCF ao uso de fármacos para aumentar o desempenho desportivo por parte dos atletas de alta competição ${ }^{10,40}$. Também se argumenta que os feitos realizados sob influência do MCF poderão ser menos valorizados, tanto social como individualmente, pois foram obtidos sem o esforço ou sacrifício necessários para os obter normalmente ${ }^{11}$. Entre os estudantes, a questão da injustiça parece ser relevante ${ }^{31}$, uma vez que os resultados obtidos por indivíduos sob MCF podem ser superiores aos de seus pares apenas pelo consumo dos nootrópicos ${ }^{3}$. Apesar deste facto, as implicações legais e médicas do uso de nootrópicos surgem como mais importantes, principalmente para não optar pelo $\mathrm{MCF}^{10}$.

\section{Consumo crescente e dependência}

Apesar de os meios de comunicação exagerarem os níveis de MCF e descreverem um aumento sem precedentes, principalmente na comunidade académica ${ }^{10,21,41}$, a verdade é que alguns estudos têm reportado um crescimento efetivo do MCF nesta população e também na comunidade científica ${ }^{3}$. No Reino Unido, o número de prescrições de fármacos nootrópicos tem aumentado contínua e inexplicavelmente de 220 mil em 1998 para 418,3 mil em $2004^{3}$. Se a isto se junta a informação de que cerca de $90 \%$ do modafinil é usado por indivíduos saudáveis (sem patologia do sono) para aumentar a vigilância e atenção ${ }^{3}$, torna-se cada vez mais claro que o consumo de nootrópicos parece ter se alastrado ao longo dos últimos anos, o que levanta a questão sobre a dependência destes produtos.

Estudos demonstram o bloqueio do transporte de dopamina exercido pelo modafinil e o consequente aumento deste neurotransmissor em zonas do encéfalo tipicamente associadas à dependência e viciação em substâncias ${ }^{3,47}$. Outros relatam que um em cada 20 utilizadores de nootrópicos sujeitos a prescrição atende a critérios diagnósticos para dependência ou abuso de substâncias ${ }^{11}$. A dependência também pode estar associada ao facto de os indivíduos se sentirem menos aptos quando não consomem estes fármacos, resultando em coação ao consumo.

\section{Discussão: futuro promissor? Ou inevitável, de facto?}

A crescente prática e a discussão em torno do MC em geral e do MCF em particular são fatores inegavelmente pertinentes para esta reflexão, uma vez que parece muito provável que o tema esteja apenas nos seus primórdios e que venha a ganhar ainda mais relevância. Falar do futuro desta prática considerando as ideias e diretrizes do presente é importante devido à miríade de questões relacionadas ao tema, bem como à falta de definição sobre que regras deverão ou não ser impostas ao MCF.

Será legalmente admissível proibi-lo em determinados contextos? Será eticamente aceitável torná-lo obrigatório em outros? Até que ponto será necessário regulamentar sua prática? Até que ponto poderemos definir a distância entre 
tratar alguém saudável e melhorar alguém com um défice de cognição? Pensar que todas estas questões terão uma resposta imediata adequada ou simples, ou mesmo uma resposta sequer, será quase duvidar da pertinência da discussão.

Antes de tudo, o MCF tem sempre de ser contextualizado no nível social e individual, pois esse contexto - seja um desejo individual para o melhoramento, seja um desejo social para que os indivíduos que constituem a sociedade (e, em consequência, a própria sociedade) tornem-se melhores é o estímulo fundamental dessa prática. O MCF pode tornar-se mais-valia importante para indivíduos em circunstâncias sociais difíceis, ou pode ser apenas mais um agravante das disparidades sociais caso só esteja ao alcance das classes mais altas. É sabido que situações sociais menos favorecidas são causadas e perpetuadas, em muitos casos, pelos problemas ou dificuldades cognitivas dos indivíduos nessas circunstâncias. Pelas suas limitações intelectuais, estes também são designados low-achievers ou underachievers ${ }^{1,3,12,28}$.

Vários estudos documentam o padrão de dose-resposta para os nootrópicos do $\mathrm{MCF}^{11}$, frequentemente descrito como curva em $U$ invertido que relaciona os níveis de dopamina com as capacidades cognitivas. Ou seja, indivíduos com níveis muito baixos ou muito altos de dopamina terão piores capacidades de cognição quando comparados a indivíduos com níveis ideais deste neurotransmissor ${ }^{11}$. Desta forma, aqueles que estão na parte ascendente da curva tendem a ter resultados muito melhores quando aplicado o MCF do que aqueles que estão colocados no meio ou já na parte descente da curva. Por exemplo, indivíduos com menor capacidade basal de memória de trabalho tendem a obter melhores resultados do que sujeitos com muito boa memória de trabalho, sendo que estes últimos podem não ser afetados ou até ser prejudicados pelo MCF.

Tudo isto torna relevante a pergunta: não será pressuposto mais justo assumir o MCF como realidade importante e necessária para estes low-achievers? Uma vez mais, isto reflete o impacto do indivíduo na sociedade que ele ajuda a constituir sociedade, não se pode esquecer, que muitas vezes o impactou em primeiro lugar quando não lhe garantiu (ou aos seus antepassados) as condições para que tivesse uma vida melhor do que aquela que tem ${ }^{34}$.
Ao presumir que há um Q.I. mínimo que proporciona a alguém a capacidade de viver uma vida melhor do que a possível abaixo desse valor, a importância do MCF para estes low-achievers é quase autoexplicativa, e a possibilidade de um futuro "promissor" para o indivíduo pode ter grandes repercussões sociais. Será justo, então, em uma sociedade tecnologicamente avançada e em que o Q.I. é fundamental para completar certo nível educacional (como atualmente), condenar um indivíduo a apenas raramente conseguir uma educação de qualidade e, com isso, a possibilidade de uma vida melhor? Haverá diferença real entre melhorar este indivíduo e tratar alguém com défice cognitivo quando esse défice é determinado apenas por um nível estabelecido pela própria sociedade? E tornar-se-á "inevitável", no futuro, proceder sempre desta maneira? A diferença entre os indivíduos ditos normais e os low-achievers é apenas um limite traçado por nós. Se essa diferença for sempre se exacerbando graças ao MCF, inevitavelmente este se tornará quase mandatório, usando fármacos atuais ou outros.

A ideia oposta a esta expõe problemática importante: a do custo do melhoramento, que prevê que o MCF (regulamentado ou não) ficaria reservado às classes sociais mais elevadas. Ou seja, indivíduos menos favorecidos seriam reféns de uma plutocracia em que apenas os mais ricos podem pagar pelo MCF, o que contribuiria para a manutenção ou o aumento das desigualdades sociais. Ou então seriam reféns de uma plutocracia em que as classes sociais mais abastadas não teriam interesse no acesso das classes desfavorecidas ao MCF, pois não veriam vantagem pessoal nisso (embora possam reconhecer o benefício social do potencial aumento de Q.I. da população) ${ }^{48}$.

A não regulamentação ou ilegalização do MCF provavelmente conduzirá ao segundo cenário, associado ainda a um mercado negro para compra e venda destes produtos e à falta de acompanhamento médico das pessoas que consomem os nootrópicos. Estes três pontos são claramente deletérios ao emprego do MCF no nível social. Não sendo fatores promissores, serão eles verdadeiramente inevitáveis?

A dualidade indivíduo-sociedade está patente em várias outras questões que o MCF tem levantado. O meio académico é, sem dúvida, o mais abordado na literatura e nos meios de 
comunicação sobre este assunto, principalmente nos Estados Unidos. Ligando este argumento ao ponto anterior, há que se notar que o meio académico estadunidense é reconhecidamente frequentado por indivíduos de classes sociais mais altas, e é principalmente nas universidades mais competitivas que se reporta a maior adesão ao $\mathrm{MCF}^{21}$.

Referiram-se anteriormente as taxas do uso do MCF, mas, mais do que isso, importa compreender que a maioria dos estudantes não considera o MCF inerentemente mau. $O$ mais que questionam é o grau de justiça dos resultados obtidos com sua ajuda, e isto depende se são estudantes que aderem ou não a esta prática. Fundamentalmente, essa parece ser a única questão ética de alguma relevância para essa população, sendo apontada quando em comparação aos grandes problemas do MCF para estes indivíduos: sua legalidade e o desconhecimento dos seus efeitos secundários e em longo prazo ${ }^{10}$. Importa frisar que os estudantes valorizam mais a livre escoIha sobre o uso do MCF do que a justiça dos resultados obtidos por parte de quem o adota.

É inegável o contexto de competição crescente das universidades e a pressão para produzir resultados. O uso do MCF parece estar mais relacionado com a pressão e o estresse do que com os resultados efetivamente obtidos. As melhorias parecem escassas e o MCF parece ser mais utilizado para suportar o estresse e para tornar as tarefas enfadonhas mais interessantes. Quem advoga seu uso admite que o MCF se torna inútil se não for acompanhado do estudo adequado, pelo que, em contexto académico e com os nootrópicos disponíveis, os seus efeitos parecem ser muito diminutos. Reconhece-se que a percepção de melhoramento pode ser superior ao melhoramento efetivo ${ }^{49}$, e este efeito placebo é também relevante.

Posto isto, é importante perceber que os fármacos mais utilizados atualmente se destinam a aumentar a capacidade de atenção e vigilância, e, em alguns casos, da memória. O uso de fármacos diferentes para melhorar outras capacidades cognitivas poderá ser, em determinados contextos, considerado absolutamente errado - por exemplo, aderir a um plano de MCF que aumente deliberadamente a criatividade em uma prova que demanda conceber uma obra. Uma questão paralela seria: fora do contexto da prova, é relevante a obra ter sido criada recorrendo-se ao MCF? O uso de outras substâncias psicotrópicas, como estimuladores, não é comumente associado à própria arte? É difícil julgar em absoluto, porque inúmeros contextos teriam de ser considerados.

Por outro lado, não raro o MCF é comparado ao uso (ilegal) de substâncias para melhorar o desempenho de atletas de alta competição. Os estudantes não parecem considerar esta comparação tão relevante ${ }^{10}$, muito porque a finalidade de uma prova competitiva é, justamente, a competição e a classificação obtida para aquele instante, enquanto, no contexto académico, o resultado de um exame ou de um conjunto de exames classifica apenas o indivíduo, apesar de sempre comparado aos seus pares, e não oferece, por si mesmo, a conclusão de todo um processo.

Isto é muito mais evidente no contexto profissional. Se tomarmos por exemplo uma cirurgia, será irrelevante para o seu resultado que o cirurgião tenha usado do MCF. Isto porque a finalidade do processo não é a competição, nem a comparação do cirurgião a outros cirurgiões, mas sim o sucesso da operação e o bem-estar do doente.

No contexto profissional há ainda outras problemáticas, principalmente em ocupações de alto risco e responsabilidade ou sujeitas a maior pressão. São paradigmáticos os exemplos do MCF associado a cirurgiões (entre outras especialidades médicas), a pilotos de avião e controladores aéreos, a militares e às forças de intervenção. Estes profissionais operam muitas vezes em situações de risco, com vidas humanas em jogo. Desta forma, tem surgido a questão de "novas obrigações" para este tipo de profissional ${ }^{26}$. Não será expectável que o estado da arte venha a recomendar aos profissionais que usem do MCF?

Esta questão se coloca porque as ditas "novas obrigações" são apenas normas em tudo semelhantes às que foram impostas no passado, de forma a obter resultados melhores ou tornar os procedimentos mais seguros. A esterilização do material cirúrgico ou a lavagem das mãos entre cirurgias (e entre consultas) não eram imposições até serem reconhecidas como medidas imprescindíveis. Será o MCF medida imprescindível (e, portanto, inevitável) em certas profissões?

Mais uma vez, a dualidade indivíduo-sociedade está evidente. A sociedade exige muitas vezes que alguns indivíduos abdiquem de algo para o benefício de todos. Militares abdicam da sua segurança e bem-estar em prol da defesa do seu país, pilotos de 
avião têm vidas familiares mais conturbadas pelas constantes viagens, médicos abdicam de horas em família e custeiam por si mesmos formações continuadas em seu já pouco tempo. Tudo isto implica uma troca entre o indivíduo e a sociedade, muitas vezes em maior benefício desta última.

Em determinadas situações, poderá ser esperado que os profissionais adiram ao MCF porque, dadas as circunstâncias, é o melhor a fazer. Poderá ser esperado que um cirurgião, após longas horas no bloco operatório, seja ética ou legalmente obrigado a tomar modafinil, por exemplo, por questão de segurança. Certos estudos admitem que isto só não acontece porque se recorre mais frequentemente à cafeína, apenas por estar disponível rapidamente e sem grandes entraves ${ }^{26}$.

Surge então o dilema neuroético de tornar obrigatório o consumo de um fármaco potencialmente alterador da cognição devido à profissão do indivíduo. Mas, admitindo-se que determinado profissional está trabalhando há longas horas e que suas capacidades cognitivas se encontram diminuídas, não se poderá compará-lo a um low-achiever? Se esse indivíduo estiver agora na parte ascendente da curva em $U$ invertido que traduz a relação entre os níveis de dopamina e a capacidade cognitiva, os fármacos do MCF servirão apenas para repor os níveis associados à normalidade para ele. Isto é semelhante ao caso dos profissionais que operam maquinaria pesada e que sofrem de epilepsia ou diabetes, que são obrigados a consumir determinado fármaco (potencialmente alterador de algumas funções cerebrais) para que não haja efeitos adversos sobre outros indivíduos. Em profissão de alto risco, um profissional exausto não estará quase na mesma situação?

Observe-se, no entanto, que no nível profissional e académico poderá a exigência aumentar desmesuradamente, mas não por um bem social. Poderá haver casos em que seja exigido o MCF aos trabaIhadores de dada empresa apenas para aumentar o lucro, e não por questões técnicas ou de segurança. Estes casos são mais perigosos porque a dita "liberdade cognitiva" (ou integridade cognitiva) não se encontra protegida legalmente da mesma forma que a integridade física ${ }^{26}$. Vários especialistas em bioética argumentam que o MCF pode colocar problemas quanto à autenticidade e personalidade dos indivíduos, podendo mesmo ameaçar o que é ser humano ${ }^{3}$. Isto é particularmente preocupante no caso de o MCF ser uma imposição profissional desregulada e com vista apenas ao lucro de determinada empresa, e não da sociedade.

Todo o anteriormente exposto depende criticamente do estudo do MCF naquilo que é o fundamento da sua ação: que efeitos os nootrópicos têm sobre o encéfalo e restante fisiologia daqueles que os consomem sendo saudáveis? A falta de estudos prospectivos (ou outros) com amostras significativas, aplicados aos diferentes nootrópicos e mesmo em diferentes contextos, tem tornado difícil discutir a temática. No caso das profissões de risco, o uso de MCF com efeitos estudados seria mais facilmente recomendado (ou mesmo tornado obrigatório) circunstancialmente, caso os efeitos secundários ou em longo prazo não fossem problemáticos.

Atualmente, estudos reportam boa tolerância aos nootrópicos, mas há casos de aumento da frequência cardíaca, pressão arterial, cefaleias, ansiedade, tonturas, náuseas e insónias com o metilfenidato e, além destes, problemas gastrointestinais, poliúria, palpitações, problemas do sono, depressão, psicose e efeitos cardiovasculares no caso do modafinil ${ }^{11}$. A cafeína tem sido, por isto e por ser um xenobiótico conhecido, mais amplamente consumida como estimulante do sistema nervoso central ${ }^{11}$ e nootrópico. Seus efeitos cerebrais estão mais bem estudados e, aparentemente, parecem ser menos amplos do que os nootrópicos sujeitos a prescrição médica ${ }^{8}$.

Finalmente, há que se mencionar o caso do MCF em crianças e adolescentes. Conforme dito, o desconhecimento dos efeitos totais desses fármacos torna sua aplicação em crianças extremamente questionável. Não sendo possível presumir seu consentimento, pode estar em causa uma interferência ilegítima no exercício futuro da autonomia desse jovem.

O facto de seu encéfalo estar em desenvolvimento deve ser considerado pela cognição em si, mas não só. A cognição não existe independentemente da moralidade ou da emoção ${ }^{48,50}$, pelo que, no caso de indivíduos que ainda estão a desenvolver facetas da personalidade e do que é ser humano, afetar este equilíbrio com o uso de fármacos colocará mesmo em causa o seu direito a um futuro aberto. Interferir neste ponto do desenvolvimento põe em questão a base do que é a condição humana, já que a própria criança acaba não tendo a plena oportunidade de a desenvolver 
e perceber. Basta lembrar que crianças com PHDA atribuem muito do seu mau comportamento às situações em que não estão medicadas.

Que se poderá esperar se mais crianças forem medicadas sem haver razão para tal? A dependência destes fármacos - ainda que não só neste caso e ainda que não necessariamente fisiológica - é problema a evitar, uma vez que vários indivíduos poderão considerar-se capazes de determinadas tarefas apenas sob a influência de fármacos. Pior será se estes indivíduos duvidarem mesmo da sua humanidade quando não estão sob o efeito dos nootrópicos.

\section{Considerações finais}

As várias questões neuroéticas, médicas e legais que o MCF envolve tornam este tema um desafio e uma preocupação. Deverá sempre considerar-se a beneficência e a não maleficência de todo o processo para o indivíduo, ponderando sua autonomia e condição. Nas crianças, o cuidado deve ser redobrado, pois pode estar em causa não apenas a limitação do exercício futuro da autonomia pessoal (direito a um futuro aberto), mas também o condicionamento da própria identidade pessoal.

Por tudo isso, é crucial o estudo mais aprofundado da farmacodinâmica de cada nootrópico, e que a regulamentação destes produtos não seja ignorada nem pela classe médica nem pela sociedade. O objetivo de melhorar a cognição foi e será sempre perseguido por este ou por outros métodos. O que mais importará compreender será até que ponto cada melhoramento, por mais promissor que seja, nos afasta da nossa noção de humanidade ou se inevitavelmente, porque é tão humano fazê-lo, nos aproxima dela.

\section{Referências}

1. Reiner M, Gruzelier J, Bamidis PD. Cognitive enhancement: a system view. Int J Psychophysiol [Internet]. 2017 [acesso 26 nov 2019];122:1-5. DOI: 10.1016/j.ijpsycho.2017.07.011

2. Roskies A. Neuroethics for the new millenium. Neuron [Internet]. 2002 [acesso 26 nov 2019];35(1):21-3. DOI: 10.1016/s0896-6273(02)00763-8

3. Mohamed AD. Neuroethical issues in pharmacological cognitive enhancement. Wiley Interdiscip Rev Cogn Sci [Internet]. 2014 [acesso 26 nov 2019];5(5):533-49. DOI: 10.1002/wcs.1306

4. Bostrom N, Roache R. Ethical issues in human enhancement. In: Ryberg J, Petersen T, Wolf C, editores. New waves in applied ethics. London: Palgrave Macmillan; 2007. p. 120-52.

5. Savulescu J, ter Meulen RHJ, Kahane G, editores. Enhancing human capacities. Chichester: WileyBlackwell; 2011.

6. Glannon W. Brain, body and mind: neuroethics with a human face. Oxford: Oxford University Press; 2011.

7. President's Council on Bioethics. Beyond therapy: biotechnology and the pursuit of happiness: a report of the President's Council on Bioethics [Internet]. Washington: The President's Council on Bioethics; 2003 [acesso 26 nov 2019]. Disponível: https://bit.ly/3clDu1d

8. Franke AG, Lieb K, Hildt E. What users think about the differences between caffeine and illicit/prescription stimulants for cognitive enhancement. PLoS One [Internet]. 2012 [acesso 26 nov 2019];7(6):e40047. DOI: 10.1371/journal.pone.0040047

9. Hildt E. Cognitive enhancement: a critical look at the current debate. In: Hildt E, Franke AG, editores. Cognitive enhancement: an interdisciplinary perspective [Internet]. Dordrecht: Springer; 2013 [acesso 26 nov 2019]. p. 1-14. DOI: 10.1007/978-94-007-6253-4_1

10. Singh I, Bard I, Jackson J. Robust resilience and substantial interest: a survey of pharmacological cognitive enhancement among university students in the UK and Ireland. PLoS One [Internet]. 2014 [acesso 26 nov 2019];9(10):e105969. DOI: 10.1371/journal.pone.0105969

11. Maslen H, Faulmuller N, Savulescu J. Pharmacological cognitive enhancement: how neuroscientific research could advance ethical debate. Front Syst Neurosci [Internet]. 2014 [acesso 26 nov 2019];8:107. DOI: $10.3389 /$ fnsys.2014.00107 
12. Tomažič T, Čelofiga AK. Ethical aspects of the abuse of pharmaceutical enhancements by healthy people in the context of improving cognitive functions. Philos Ethics Humanit Med [Internet]. 2019 [acesso 26 nov 2019];14:7. DOI: 10.1186/s13010-019-0076-5

13. Dresler M, Sandberg A, Ohla K, Bublitz C, Trenado C, Mroczko-Wasowicz A et al. Non-pharmacological cognitive enhancement. Neuropharmacology [Internet]. 2013 [acesso 26 nov 2019];64:529-43. DOI: 10.1016/j.neuropharm.2012.07.002

14. Mohamed AD, Sahakian BJ. The ethics of elective psychopharmacology. Int J Neuropsychopharmacol [Internet]. 2012 [acesso 26 nov 2019];15(4):559-71. DOI: 10.1017/S146114571100037X

15. Coveney CM, Nerlich B, Martin P. Modafinil in the media: metaphors, medicalisation and the body. Soc Sci Med [Internet]. 2009 [acesso 26 nov 2019];68(3):487-95. DOI: 10.1016/j.socscimed.2008.11.016

16. Sattler S. Nonmedical use of prescription drugs for cognitive enhancement as response to chronic stress especially when social support is lacking. Stress Health [Internet]. 2019 [acesso 26 nov 2019];35(2):127-37. DOI: $10.1002 /$ smi.2846

17. Hall WD, Lucke JC. The enhancement use of neuropharmaceuticals: more scepticism and caution needed. Addiction [Internet]. 2010 [acesso 26 nov 2019];105(12):2041-3. DOI: 10.1111/j.1360-0443.2010.03211.x

18. Advokat $\mathrm{C}$. What are the cognitive effects of stimulant medications? Emphasis on adults with attentiondeficit/hyperactivity disorder (ADHD). Neurosci Biobehav Rev [Internet]. 2010 [acesso 26 nov 2019];34(8):1256-66. DOI: 10.1016/j.neubiorev.2010.03.006

19. Savulich G, Piercy T, Bruhl AB, Fox C, Suckling J, Rowe JB, Sahakian BJ. Focusing the neuroscience and societal implications of cognitive enhancers. Clin Pharmacol Ther [Internet]. 2017 [acesso 26 nov 2019];101(2):170-2. DOI: $10.1002 / c p t .457$

20. Bruhl AB, Sahakian BJ. Drugs, games, and devices for enhancing cognition: implications for work and society. Ann N Y Acad Sci [Internet]. 2016 [acesso 28 jan 2021];1369(1):195-217. DOI: 10.1111/nyas.13040

21. Vagwala MK, Bicquelet A, Didziokaite G, Coomber R, Corrigan O, Singh I. Towards a moral ecology of pharmacological cognitive enhancement in British Universities. Neuroethics [Internet]. 2017 [acesso 26 nov 2019];10(3):389-403. DOI: 10.1007/s12152-017-9336-5

22. Wilens TE, Adler LA, Adams J, Sgambati S, Rotrosen J, Sawtelle R et al. Misuse and diversion of stimulants prescribed for ADHD: a systematic review of the literature. J Am Acad Child Adolesc Psychiatry [Internet]. 2008 [acesso 26 nov 2019];47(1):21-31. DOI: 10.1097/chi.0b013e31815a56f1

23. McCabe SE, Knight JR, Teter CJ, Wechsler H. Non-medical use of prescription stimulants among US college students: prevalence and correlates from a national survey. Addiction [Internet]. 2005 [acesso 26 nov 2019];100(1):96-106. DOI: 10.1111/j.1360-0443.2005.00944.x

24. Arria AM, Caldeira KM, O'Grady KE, Vincent KB, Johnson EP, Wish ED. Nonmedical use of prescription stimulants among college students: associations with attention-deficit-hyperactivity disorder and polydrug use. Pharmacotherapy [Internet]. 2008 [acesso 29 jan 2021];28(2):156-69. DOI: 10.1592/phco.28.2.156

25. McCabe SE, West BT, Teter CJ, Boyd CJ. Trends in medical use, diversion, and nonmedical use of prescription medications among college students from 2003 to 2013: connecting the dots. Addict Behav [Internet]. 2014 [acesso 26 nov 2019];39(7):1176-82. DOI: 10.1016/j.addbeh.2014.03.008

26. Santoni de Sio F, Faulmuller N, Vincent NA. How cognitive enhancement can change our duties. Front Syst Neurosci [Internet]. 2014 [acesso 26 nov 2019];8:131. DOI: 10.3389/fnsys.2014.00131

27. Bostrom N, Sandberg A. Cognitive enhancement: methods, ethics, regulatory challenges. Sci Eng Ethics [Internet]. 2009 [acesso 26 nov 2019];15(3):311-41. DOI: 10.1007/s11948-009-9142-5

28. Jotterand F. Childhood brain development, the educational achievement gap, and cognitive enhancement. Front Pharmacol [Internet]. 2018 [acesso 26 nov 2019];9:1142. DOI: 10.3389/fphar.2018.01142

29. Glannon W. Psychopharmacology and memory. J Med Ethics [Internet]. 2006 [acesso 26 nov 2019];32(2):74-8. DOI: $10.1136 /$ jme.2005.012575 
30. Farah MJ, Illes J, Cook-Deegan R, Gardner H, Kandel E, King P et al. Neurocognitive enhancement: what can we do and what should we do? Nat Rev Neurosci [Internet]. 2004 [acesso 26 nov 2019];5(5):421-5. DOI: $10.1038 / \mathrm{nrn} 1390$

31. Faber NS, Savulescu J, Douglas T. Why is cognitive enhancement deemed unacceptable? The role of fairness, deservingness, and hollow achievements. Front Psychol [Internet]. 2016 [acesso 26 nov 2019];7:232. DOI: 10.3389/fpsyg.2016.00232

32. Colaneri N, Sheldon M, Adesman A. Pharmacological cognitive enhancement in pediatrics. Curr Opin Pediatr [Internet]. 2018 [acesso 26 nov 2019];30(3):430-7. DOI: 10.1097/MOP.0000000000000615

33. Krutzinna J. Can a welfarist approach be used to justify a moral duty to cognitively enhance children? Bioethics [Internet]. 2016 [acesso 26 nov 2019];30(7):528-35. DOI: 10.1111/bioe.12244

34. Dunlop M, Savulescu J. Distributive justice and cognitive enhancement in lower, normal intelligence. Monash Bioeth Rev [Internet]. 2014 [acesso 26 nov 2019];32(3-4):189-204. DOI: 10.1007/ s40592-014-0014-7

35. Garasic MD, Lavazza A. Moral and social reasons to acknowledge the use of cognitive enhancers in competitive-selective contexts. BMC Med Ethics [Internet]. 2016 [acesso 26 nov 2019];17:18. DOI: 10.1186/ s12910-016-0102-8

36. DeGrazia D. Enhancement technologies and human identity. J Med Philos [Internet]. 2005 [acesso 26 nov 2019];30(3):261-83. DOI: 10.1080/03605310590960166

37. Pickersgill M, Hogle L. Enhancement, ethics and society: towards an empirical research agenda for the medical humanities and social sciences. Med Humanit [Internet]. 2015 [acesso 26 nov 2019];41(2):136-42. DOI: 10.1136/medhum-2015-010718

38. Horn G, Barnes J, Brownsword R, Deakin JFW, Gilmore I, Hickman M et al. Brain science, addiction and drugs: an Academy of Medical Sciences working group report chaired by Professor Sir Gabriel Horn FRS FRCP. London: Academy of Medical Sciences; 2008.

39. Weiss B. Vulnerability of children and the developing brain to neurotoxic hazards. Environ Health Perspect [Internet]. 2000 [acesso 26 nov 2019];108(supl 3):375-81. DOI: 10.1289/ehp.00108s3375

40. Chandramouleeswaran S, Edwin NC, Rajaleelan W. Dealing with requests for pharmacological cognitive enhancement from healthy students. Indian J Med Ethics [Internet]. 2016 [acesso 26 nov 2019];1(3):196. DOI: 10.20529/JMME.2016.058

41. Lucke J, Jensen C, Dunn M, Chan G, Forlini C, Kaye S et al. Non-medical prescription stimulant use to improve academic performance among Australian university students: prevalence and correlates of use. BMC Public Health [Internet]. 2018 [acesso 26 nov 2019];18:1270. DOI: 10.1186/s12889-018-6212-0

42. Maher B. Poll results: look who's doping. Nature [Internet]. 2008 [acesso 26 nov 2019];452(7188):674-5. DOI: 10.1038/452674a

43. Franke AG, Roser P, Lieb K, Vollmann J, Schildmann J. Cannabis for cognitive enhancement as a new coping strategy? Results from a survey of students at four universities in Germany. Subst Use Misuse [Internet]. 2016 [acesso 26 nov 2019];51(14):1856-62. DOI: 10.1080/10826084.2016.1200619

44. Tannock R, Schachar R. Methylphenidate and cognitive perseveration in hyperactive children. J Child Psychol Psychiatry [Internet]. 1992 [acesso 26 nov 2019];33(7):1217-28. DOI: 10.1111/ j.1469-7610.1992.tb00940.x

45. DeSantis A, Noar SM, Webb EM. Speeding through the frat house: a qualitative exploration of nonmedical ADHD stimulant use in fraternities. J Drug Educ [Internet]. 2010 [acesso 26 nov 2019];40(2):157-71. DOI: 10.2190/DE.40.2.d

46. Pohl S, Boelsen H, Hildt E. Moral attitudes toward pharmacological cognitive enhancement (PCE): differences and similarities among Germans with and without PCE experience. Front Pharmacol [Internet]. 2018 [acesso 26 nov 2019];9:1451. DOI: 10.3389/fphar.2018.01451

47. Volkow ND, Fowler JS, Wang GJ, Swanson JM. Dopamine in drug abuse and addiction: results from imaging studies and treatment implications. Mol Psychiatry [Internet]. 2004 [acesso 26 nov 2019];9(6):557-69. DOI: $10.1038 /$ sj.mp.4001507 
48. Cirkovic MM. Enhancing a person, enhancing a civilization: a research program at the intersection of bioethics, future studies, and astrobiology. Camb Q Healthc Ethics [Internet]. 2017 [acesso 26 nov 2019];26(3):459-68. DOI: 10.1017/S0963180116001134

49. Ilieva I, Boland J, Farah MJ. Objective and subjective cognitive enhancing effects of mixed amphetamine salts in healthy people. Neuropharmacology [Internet]. 2013 [acesso 26 nov 2019];64:496-505. DOI: 10.1016/ j.neuropharm.2012.07.021

50. Pavarini G, McKeown A, Singh I. Smarter than thou, holier than thou: the dynamic interplay between cognitive and moral enhancement. Front Pharmacol [Internet]. 2018 [acesso 26 nov 2019];9:1189. DOI: 10.3389/fphar.2018.01189

Rafael Cardoso Oliveira - Mestre - rafaelco23@gmail.com

(D) 0000-0003-3323-4084

Rui Nunes - Doutor - ruinunes@med.up.pt

(D) 0000-0002-1377-9899

Correspondência

Rafael Cardoso Oliveira - Rua Professora Ofélia da Cruz Costa, 174, Lavra Matosinhos CEP 4455-137. Porto, Portugal.

Participação dos autores

Recebido: 24.7.2019

Rafael Cardoso Oliveira realizou a pesquisa e elaborou o texto. Rui Nunes orientou o projeto e revisou o texto. 